\title{
Harmonic Analysis and Practical Implementation of a Two-Phase Microgrid System
}

\author{
M. Alibeik*, E. C. dos Santos Jr.*, Y. Yang**, X. Wang** and F. Blaabjerg** \\ *Purdue School of Engineering and Technology \\ Indiana University-Purdue University Indianapolis \\ Indianapolis, IN \\ **Department of Energy Technology, Aalborg University, Denmark \\ e-mail: malibeik@iupui.edu, eudossan@iupui.edu, yoy@et.aau.dk, xwa@et.aau.dk,fbl@et.aau.dk
}

\begin{abstract}
This paper analyzes the harmonic contents of a non-linear load connected to a two-phase microgrid system. Although having the same harmonic content as the single-phase power system when supplying a non-linear load under balanced conditions, the two-phase microgrid system presents the following advantages: 1) constant power through the power line at the balanced condition; 2) two voltages i.e., line-to-line and phase voltages, available by using a three wire system; 3) optimized voltage utilization compared to a three-phase system; and 4) a direct connection of both symmetrical two-phase and single-phase electrical machines. This paper presents an approach for analyzing the harmonics of a two-phase non-linear load in a balanced and unbalanced cases. The mathematical model for the symmetrical component of an unbalanced two-phase system has also been presented in this paper. Finally, a practical implementation of the twophase system has been performed, where different types of loads are connected to the two-phase power line to test the voltage control performance.
\end{abstract}

\section{INTRODUCTION}

The IEC 62257-9 specifies the general requirements for implementation and design of microgrids used in the decentralized rural electrification. The microgrids covered by this recommendation are low ac voltage with the capacity less than or equal to $100 \mathrm{kVA}$. They are typically powered by a single micro power plant and do not include any voltage transformation.

In the modern scenario, the utility grid is supposed to guarantee a proper load management, demand side management, as well as to use the market price of electricity and forecasting of energy (e.g., based on wind and solar renewable sources) in order to optimize the whole distribution system [1]. An electric power system requires high efficiency, high reliability, good quality of service and high level of security in its optimized operation [2]. Those objectives can be obtained in the new power distribution systems through advances in control, communication and information technology, as well as an extensive use of power electronics. Notice that the power line type is not necessarily defined anymore by a centralized generation unit that delivers the same type of energy to all loads connected to it. Now the power distribution process goes through power electronic devices that allow more flexibility regarding how the electric link is defined.

Fig. 1 shows a microgrid being fed by a strong energy source as well as weak energy sources, (e.g., residential PhotoVoltaic (PV) and wind source). Loads are also spread throughout the line. In respect to the connection of such a microgrid, research efforts have been developed towards: 1) dc microgrid [3], 2) single-phase $60 \mathrm{~Hz}$ ac microgrid [4], 3) single-phase high-frequency ac microgrid [5], 4) three-phase three-wire microgrid [6], 5) three-phase four-wire microgrid [7], and 6) more recently the two-phase microgrid with voltages in quadrature [8], which is the topic further analyzed in this paper. The proposed two-phase microgrid presents the following advantages: 1) constant power at balanced conditions, instead of a pulsing power in the single-phase microgrid; 2) two voltages available by using a three-wire system, i.e., line-line $\left(V_{l l}\right)$ and phase $\left(V_{p h}\right)$ voltages with $V_{l l}=\sqrt{2} V_{p h}$ in contrast to the four-wire three-phase system; 3 ) lower voltage fluctuation as compared to the single-phase system at the dclink voltage of a Voltage Source Inverter (VSI) fed drive; and 4) a direct connection of both symmetrical two-phase and single-phase electrical machines. A practical implementation of the two-phase system has been performed, where different types of loads are connected to the two-phase power line to test the voltage control performance in terms of harmonic mitigations of the voltage generated by the power line. Also, experimental results validate the theoretical study showing the similarity of the current harmonic contents between the singleand two-phase micro-grids.

\section{CURrent Harmonic Analysis For a Non-Linear LOAD}

Non-linear loads inject significant current harmonics to the power network with potential harmful impacts on the power system. These harmonics should be predictable to determine how they will interact with other components in the power system. In this section, the single-phase, two-phase, and threephase rectifiers have been analyzed in terms of harmonic contents. Figs. 2(a), 2(b), and 2(c) show the single-phase, twophase, and three-phase rectifiers, respectively.

Fig. 3(a) shows the input voltage $v_{s}$, current $i_{s}$ and the dclink voltage $V_{o}$ for the single-phase rectifier. Fig. 3(b) shows 


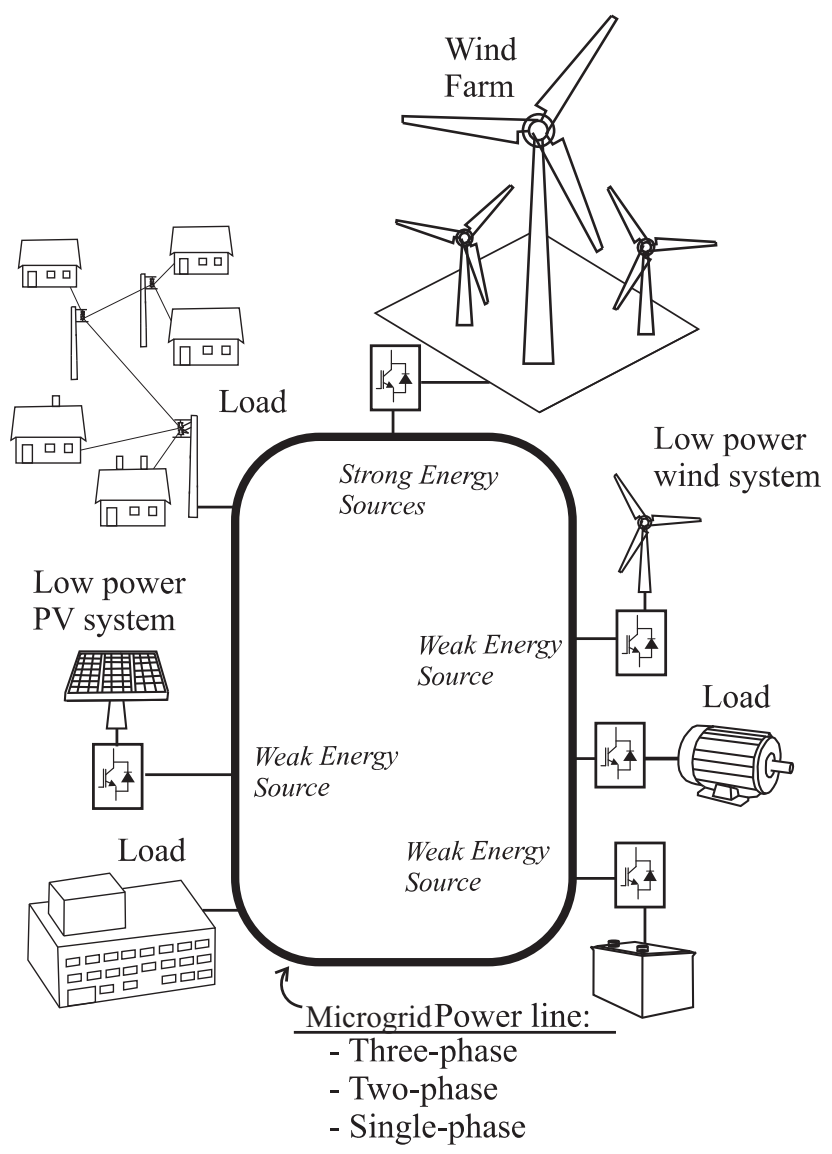

Fig. 1. Microgrid environment, where the physical electrical link with many power electronics interfaced loads is highlighted.

the line-line voltages $v_{\alpha \beta}$ and $v_{\beta \alpha}$, currents $i_{\alpha}$ and $i_{\beta}$ and the dc-link voltage $V_{o}$ for the two-phase rectifier. Fig. 3(c), in turn, shows six line-line voltages $\left(v_{a b}, v_{b a}, v_{a c}, v_{c a}, v_{b c}\right.$, and $\left.v_{c b}\right)$, the dc-link voltage $V_{o}$ and the currents $i_{a}, i_{b}$, and $i_{c}$ for the three-phase rectifier.

The terms $\gamma$ and $\eta$ in Fig. 3 show the instant at which the conduction will begin and the conduction interval, respectively. According to [9] and [10], it is possible to analyze the harmonics of single-phase and three-phase rectifiers based on the Fast Time Domain Method and the Sampled-Data Model from $\gamma$ and $\eta$. For the two-phase diode rectifier under balanced conditions, there is no current going through the neutral line. Notice from Fig. 4(a) that the phase voltages are always smaller than the line-line voltage. As a consequence, the diodes connecting to the neutral line will be off, and thus the current through the neutral line will be null. Fig. 4(b) shows the equivalent circuits of a two-phase rectifier shown in Fig. 2(b).

Since for the balanced case the neutral current is zero, the two-phase diode rectifier will have the same harmonic contents as the single phase rectifier but with different voltage amplitude. Fig. 5(a) and Fig. 5(b) are showing the current harmonic contents obtained with PSIM for single- and twophase circuits, respectively.

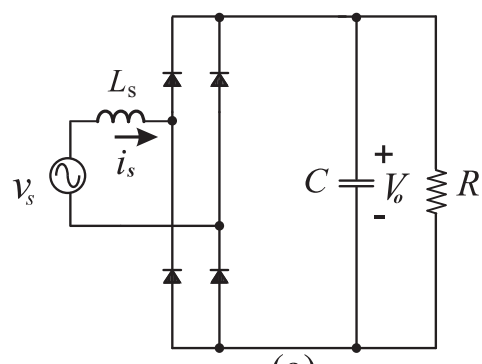

(a)

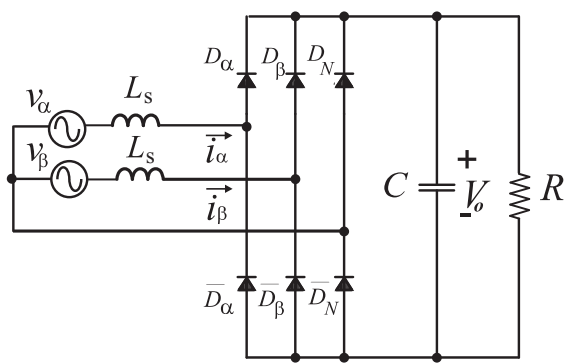

(b)

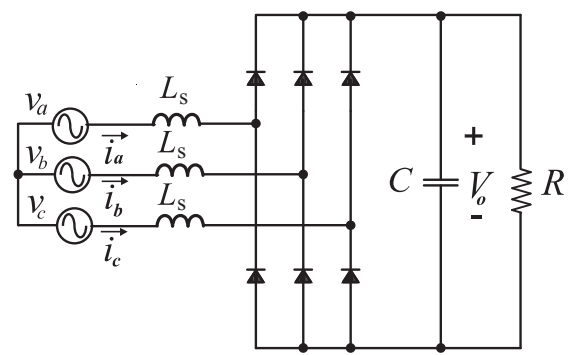

(c)

Fig. 2. Load types in microgrid: (a) Single-phase rectifier. (b) Two-phase rectifier. (c) Three-phase rectifier.

\section{Analytical Model of the Two-Phase NON-LINEAR LOAD}

This section has analyzed three different scenarios for the two-phase diode bridge rectifier: : 1) balanced, 2) unbalanced with phase displacement different from 90 degrees but with the same amplitude, and 3) unbalanced with both phase displacement different from 90 degrees and with different amplitude. Fig. 2(b) shows the two-phase diode bridge rectifier.

\section{A. Case I-Balanced System}

The first scenario is the balanced system in which two voltage sources are equal in terms of amplitude with a $90^{\circ}$ phase difference. Kirchhoff's voltage law (KVL) and Kirchhoff's current law (KCL) are used in the equivalent circuit of Fig. 4(b) leading to (1) and (2). As described in the previous section, when there is no current in the neutral line, then the system will act as a single-phase system.

$$
v_{s}(\omega t)=L_{s}^{\prime} \frac{d i_{\alpha}(\omega t)}{d(\omega t)}+V_{o}(\omega t)
$$

where $v_{s}(\omega t)=v_{\alpha}(\omega t)-v_{\beta}(\omega t)$ and $L_{s}^{\prime}=2 L_{s}$. 

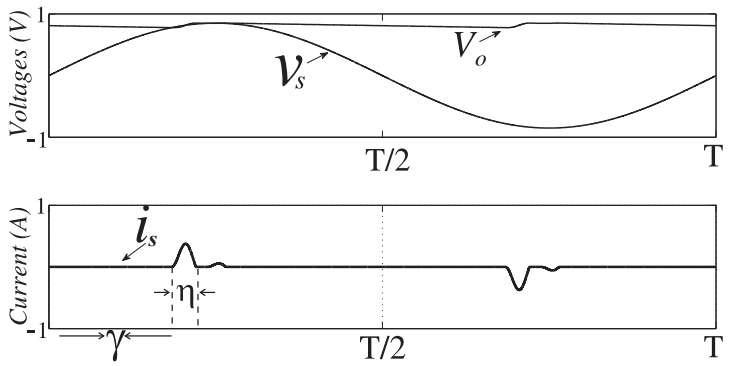

(a)
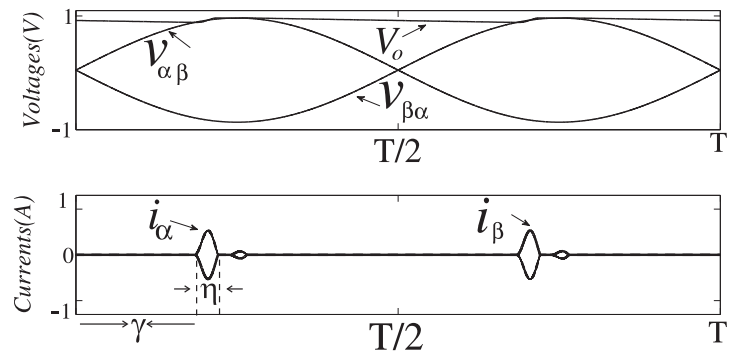

(b)
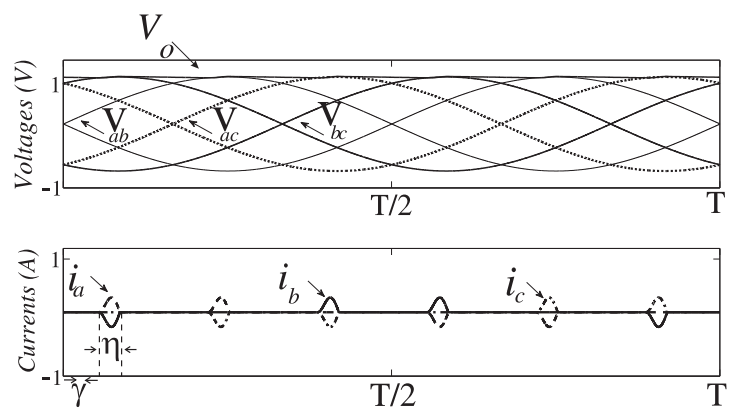

(c)

Fig. 3. Main normalized waveforms : (a) the single-phase, (b) the two-phase, and (c) the three-phase rectifiers.

Using KCL in node A of Fig. 4(b) leads to:

$$
i_{\alpha}(\omega t)=C \frac{d V_{o}(\omega t)}{d(\omega t)}+\frac{V_{o}(\omega t)}{R}
$$

where $i_{\alpha}=-i_{\beta}$.

Equations (1) and (2) constitute a system of differetial equations in terms of derivatives of the output voltage $\left(V_{o}\right)$ and the input current $\left(i_{\alpha}\right)$ which was solved using MATLAB with the syntax shown below:

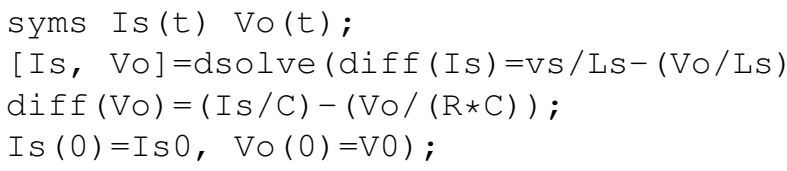

where $I_{s}$ is the same as $i_{\alpha}(\omega t)$, and $v_{s}=v_{\alpha}-v_{\beta}$

Using this syntax in MATLAB, it is possible to derive the expression for $i_{\alpha}(\omega t)$. For analyzing the harmonic contents of $i_{\alpha}(\omega t)$, the harmonic spectrum of this function should be plotted. The FFT command (Fast Fourier Transform) in MATLAB has been used in order to plot the harmonic spectrum of $i_{\alpha}(\omega t)$.

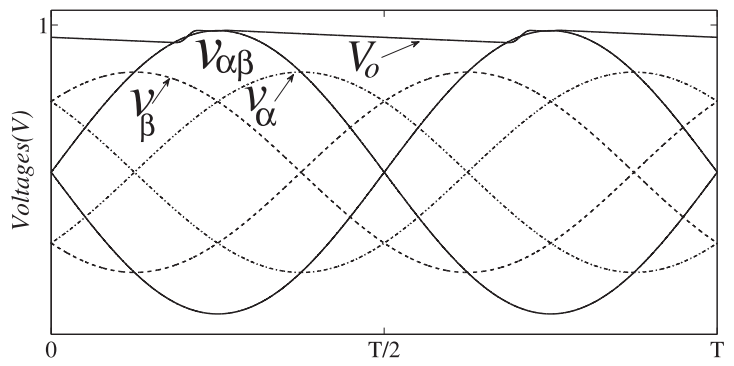

(a)
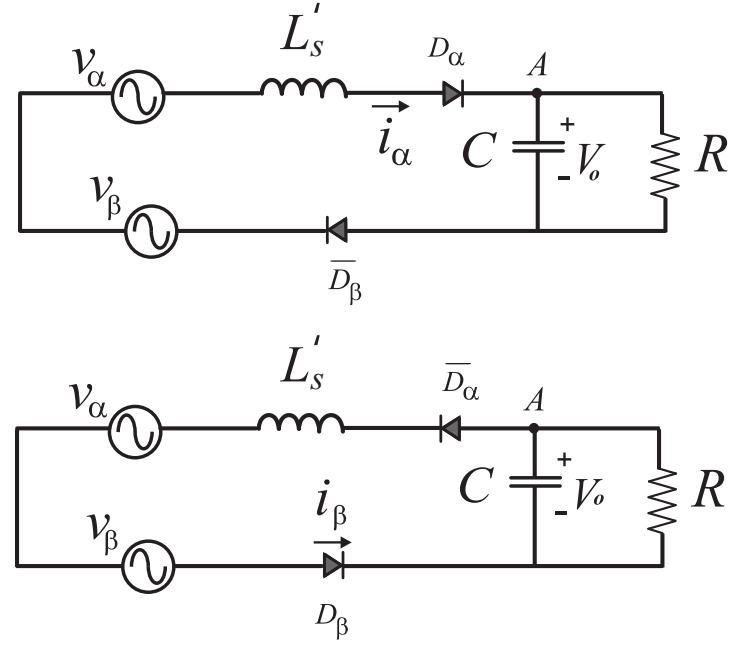

(b)

Fig. 4. (a) Line-line, phase, and output voltage for the two-phase system. (b) Equivalent model for Fig. 2(b) in a balanced case (top: from $\gamma$ to $\gamma+\eta$, bottom: from $\gamma+\frac{T}{2}$ to $\gamma+\frac{T}{2}+\eta$ ).

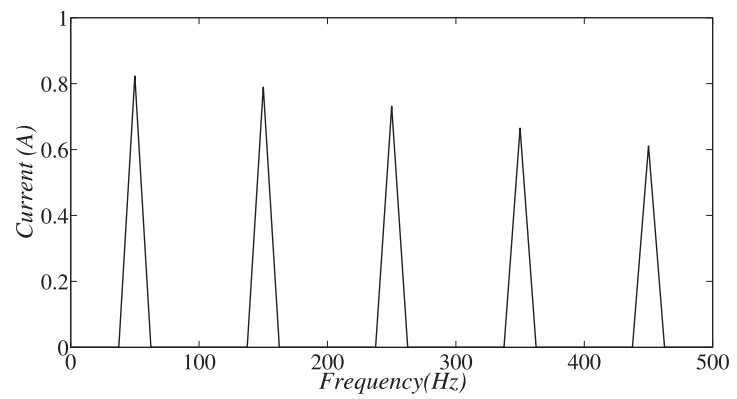

(a)

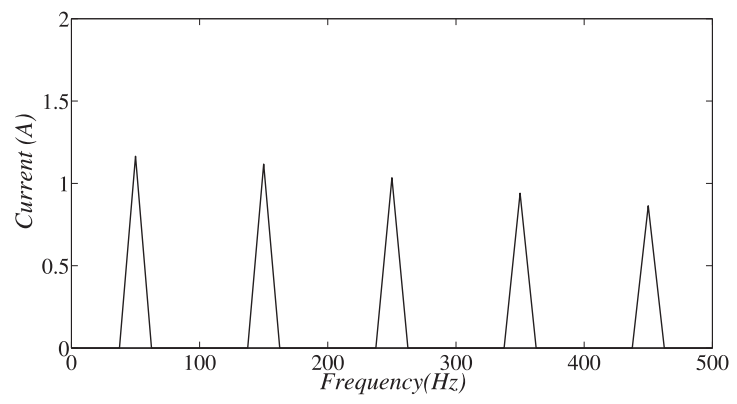

(b)

Fig. 5. Current harmonics for the: (a) single-phase, and (b) two-phase rectifiers. 
TABLE I

COMPARISON BETWEEN THE SIMULATION AND ANALYTICAL MODEL OF A TWO-PHASE DIODE BRIDGE RECTIFIER IN TERMS OF FUNDAMENTAL AND HARMONIC COMPONENTS.

\begin{tabular}{|c|c|c|}
\hline$n$ & Analytical Model & Simulation \\
\hline 1 & 1.33 & 1.30 \\
\hline 3 & 1.20 & 1.18 \\
\hline 5 & 1.12 & 1.15 \\
\hline 7 & 0.90 & 0.91 \\
\hline 9 & 0.81 & 0.75 \\
\hline
\end{tabular}

Table I shows comparison between the harmonic components derived from simulation, using PSIM, and analytically using MATLAB. In Table I, $n$ represents the number of harmonic. Each number in Table I represents the magnitude of the harmonic spectrum for that specific component. In Table I the specifications of the load are as shown below:

$$
R=395 \Omega \text {, and } C=235 \mu F \text {. }
$$

\section{B. Case II-Unbalanced System in Terms of Phase Difference}

In this section both voltages available in the two-phase diode bridge rectifier are not in quadrature. They have the same amplitude but with an angle $\theta$ other than $90^{\circ}$ as shown in Fig. 6. Where $\theta$ is the angle indicating an unbalanced system. Note that for a balanced system, $\theta$ is equal to $90^{\circ}$.

When the voltages in a two-phase diode bridge rectifier system are not in quadrature, it can unbalance the system, and as a consequence the neutral line will carry a current not equal to zero. Fig. 7(a) shows the line-line, phase, and dc link voltages in the case that voltages $v_{\alpha}(\omega t)$ and $v_{\beta}(\omega t)$ are not in quadrature. The current exists in the neutral line whenever the phase voltage is larger than the line-line voltage, as shown in Fig. 7(a). The neutral current will be different from zero when:

$$
\left|v_{\alpha \beta}\right| \leq\left|v_{\beta}\right|
$$

The range of the phase difference in which the current exists in the neutral line has been calculated as following: If the amplitude of $v_{\alpha}$ and $v_{\beta}$ is $V_{R}$, where $V_{R}$ is the rated voltage under balanced conditions, and considering Fig. 6, it yields:

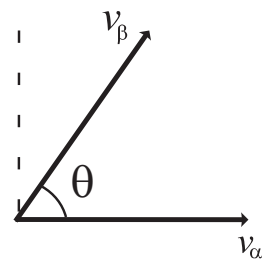

Fig. 6. Voltages with the same amplitudes and not in quadrature in the two-phase system.
Using (4) and (5) to satisfy (3) leads to an angle range of $0 \leq \theta \leq \pi / 3$. This means that whenever the phase difference of two voltages $v_{\alpha}$ and $v_{\beta}$ is in the range of $[0-\pi / 3]$ there is a neutral current. The equivalent circuit in this case is shown in Fig. 7(b).

The same calculation can be done for case I, which is also valid for this case and will result the following equations:

Using KVL in the mesh constituting $D_{\alpha}$ or $D_{N}$ and $\bar{D}_{\alpha}$ or $\bar{D}_{N}$ leads to:

$$
v_{s}(\omega t)+L_{s} \frac{d i_{N}(\omega t)}{d(\omega t)}+V_{o}(\omega t)=0
$$

in which $v_{s}(\omega t)$ is equal to $v_{\alpha}(\omega t)$ or $v_{\beta}(\omega t)$

Using KCL in node A leads to:

$$
i_{N}(\omega t)+C \frac{d V_{o}(\omega t)}{d(\omega t)}+\frac{V_{o}(\omega t)}{R}=0
$$

In Fig. 7(b) $i_{\alpha}$ and $D_{\alpha}$ can also be $i_{\beta}$ and $D_{\beta}$.

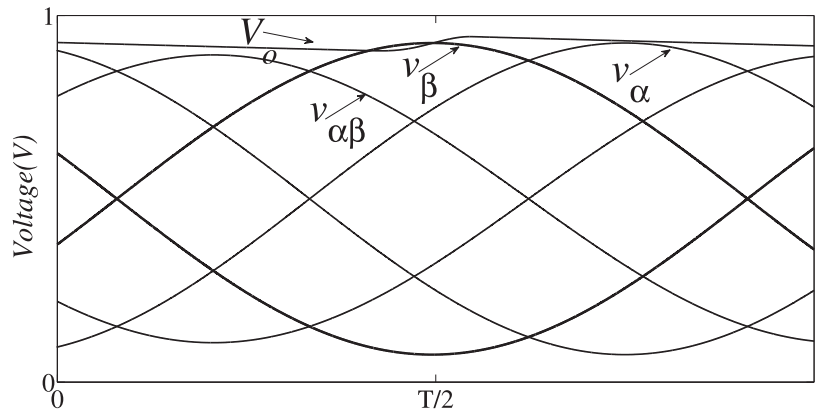

(a)
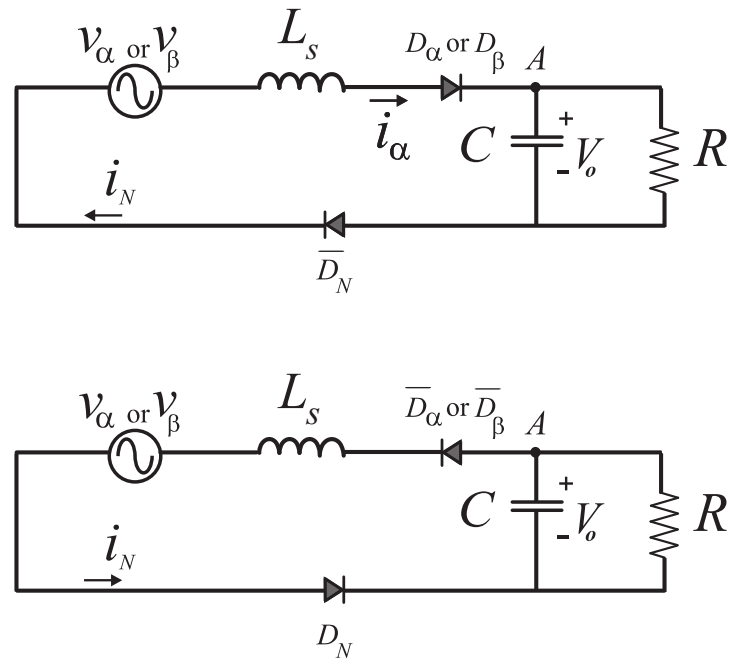

(b)

Fig. 7. Unbalanced System in Terms of Phase Difference : (a) Lineline, phase, and output voltage for a two-phase system with voltages not in quadrature, and (b) Equivalent system for case II.

\section{Case III- Unbalanced System Consisting of Voltages with Different Amplitudes and not in Quadrature}

$$
\begin{aligned}
& v_{\alpha}=V_{R} \\
& v_{\beta}=V_{R} \cos (\theta)+j V_{R} \sin (\theta)
\end{aligned}
$$

In this section a case has been discussed in which the voltages in a two-phase diode bridge rectifier system have different 
amplitudes and their phase angles are not in quadrature as shown in Fig. 8. If considering the amplitude of $v_{\alpha}$ and $v_{\beta}$ as $V_{R}$ and $K V_{R}$ respectively, then according to Fig. 8:

$$
\begin{aligned}
& v_{\alpha}=V_{R} \\
& v_{\beta}=K V_{R} \cos (\theta)+j K V_{R} \sin (\theta)
\end{aligned}
$$

where $\mathrm{K}$ is an index that represents an unbalanced system in terms of amplitude. Notice that for a balanced case, $\mathrm{K}$ is equal to 1 , The level of unbalance that causes neutral current is defined by (3) and considering the conditions (8) and (9), it leads to:

$$
P=K^{2}-2 K \cos (\theta) \leq 0
$$

in which $P(P$ is a variable that indicates the conditions whereas the neutral current will exist for the case where the DC-link capacitor voltage ripple is zero) should be less than or equal to zero to have a neutral current.

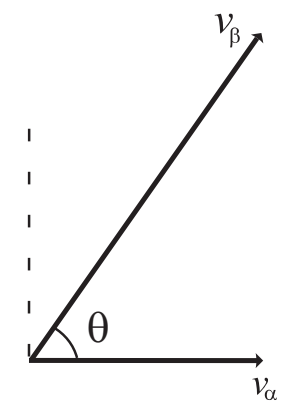

Fig. 8. Voltages with different amplitudes and not in quadrature.

Fig. 9 is showing the relationship between different values of $K, \theta$, and $P$ in (10). In Fig. 9 for any value of $K$, and $\theta$ that $P$ is negative, there exists neutral current going through the neutral line.

\section{Symmetrical Components}

The unbalanced phasors of a two-phase system can be resolved into a two balanced system of phasors using their symmetrical sets of components. The symmetrical set of components in a two-phase system are positive and negative components. The positive sequence of the components consist of two equal phasors in terms of magnitude that are in quadrature in terms of angle. These two phasors have the same phase sequences as the original phasors. The negative sequence components also consist of two equal phasors in terms of magnitude and in quadrature in terms of angle. These two phasors have the opposite phase sequences from the original phasors.

If $X_{\alpha}$ and $X_{\beta}$ are two variables (e.g., voltage or current) of the unbalanced two-phase system, then from the above descriptions it can be concluded that:

$$
\begin{aligned}
& X_{\alpha}=X_{\alpha}^{(+)}+X_{\alpha}^{(-)} \\
& X_{\beta}=X_{\beta}^{(+)}+X_{\beta}^{(-)}
\end{aligned}
$$

In (11) and (12) the superscript (+) refers to the positive component and the superscript (-) refers to the negative component of the phasors.

Fig. 10 summarizes the process to obtain the positive and negative components of the two-phase system. In this figure
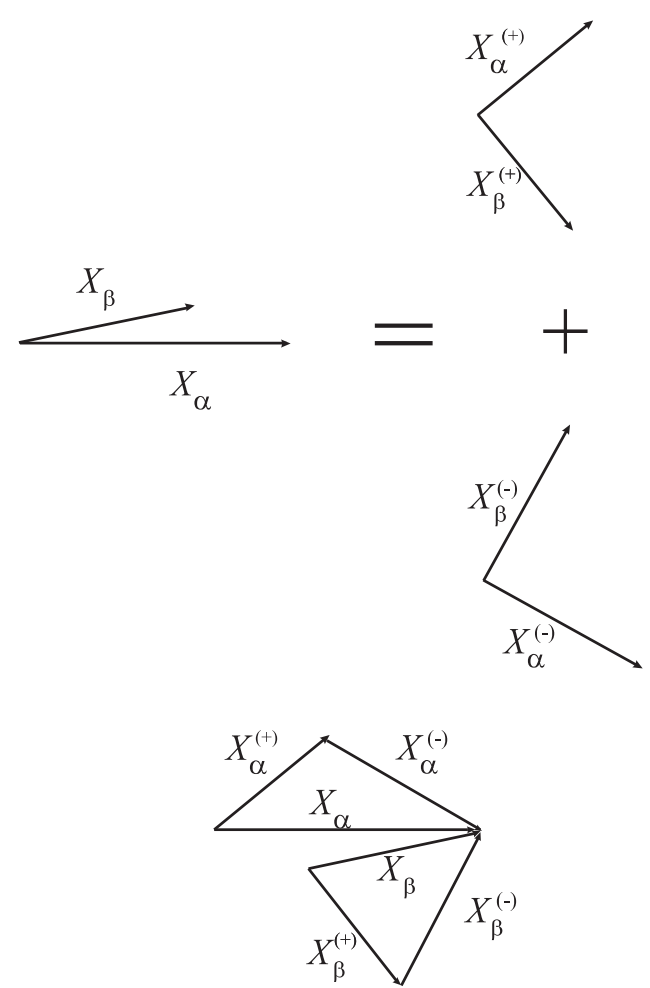

Fig. 10. The process of modeling an unbalanced two-phase system using its symmetrical components.

it is obvious that each of the unbalanced vectors is the sum of its symmetrical components as it has been shown in (11) and (12). For writing the symmetrical components in terms of the unbalanced phasors, first each component of $X_{\beta}$ can be written as the product of a component of $X_{\alpha}$ and $j=1 \angle 90^{\circ}$.

$$
\begin{gathered}
X_{\beta}^{(+)}=-j X_{\alpha}^{(+)} \\
X_{\beta}^{(-)}=j X_{\alpha}^{(-)}
\end{gathered}
$$

Substituting (11) - (12) into (13) - (14) leads to :

$$
\begin{array}{r}
X_{\alpha}=X_{\alpha}^{(+)}+X_{\alpha}^{(-)} \\
X_{\beta}=-j X_{\alpha}^{(+)}+j X_{\alpha}^{(-)}
\end{array}
$$

It can be concluded from (15) and (16) that:

$$
\left[\begin{array}{l}
X_{\alpha} \\
X_{\beta}
\end{array}\right]=A\left[\begin{array}{c}
X_{\alpha}^{(+)} \\
X_{\alpha}^{(-)}
\end{array}\right]
$$

where $A=\left[\begin{array}{cc}1 & 1 \\ -j & j\end{array}\right]$

From (17) it is obvious that:

$$
\left[\begin{array}{l}
X_{\alpha}^{(+)} \\
X_{\alpha}^{(-)}
\end{array}\right]=A^{-1}\left[\begin{array}{l}
X_{\alpha} \\
X_{\beta}
\end{array}\right]
$$




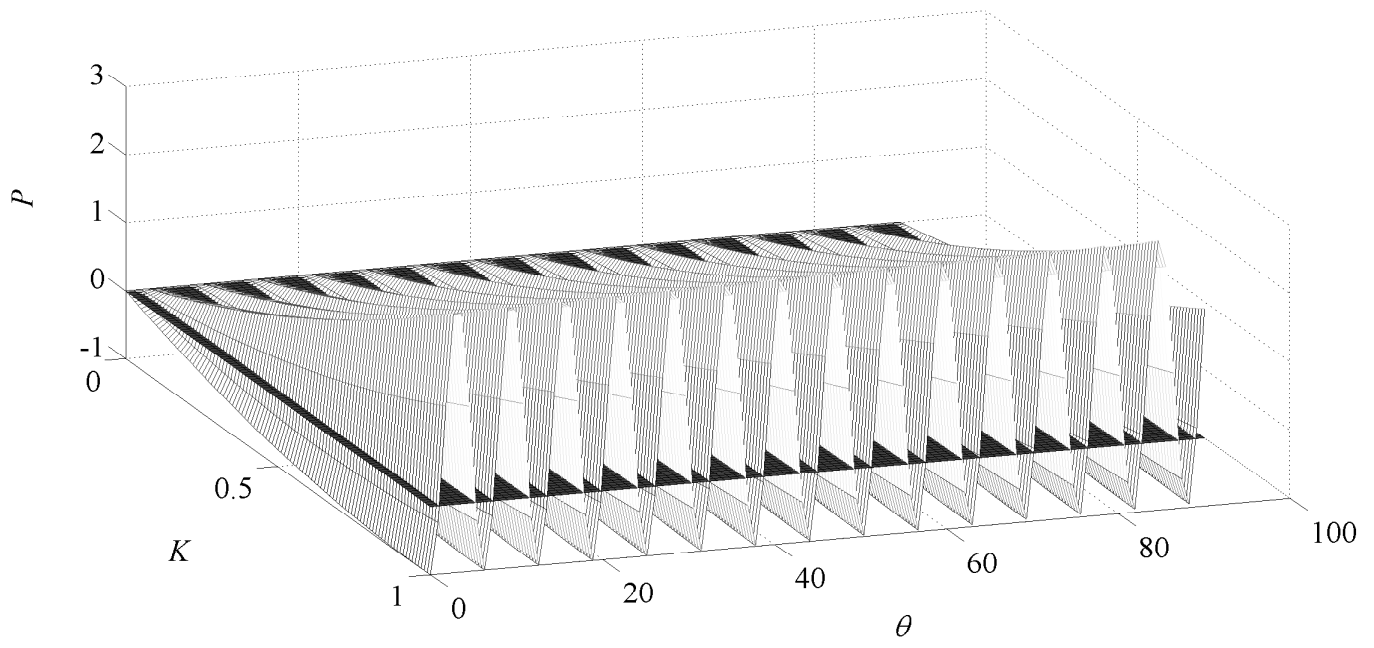

Fig. 9. Relationship between $\mathrm{K}, \theta$, and $\mathrm{P}$

where $\quad A^{-1}=\left[\begin{array}{cc}\frac{1}{2} & \frac{1 j}{2} \\ \frac{1}{2} & \frac{-1 j}{2}\end{array}\right]$

The process for the symmetrical component of $X_{\beta}$ is similar to the above process with this difference that each component of $X_{\alpha}$ should be written as a product of a component of $X_{\beta}$ with $j=1 \angle 90^{\circ}$.

$$
\begin{gathered}
X_{\alpha}^{(+)}=\frac{1}{2} X_{\alpha}+\frac{1}{2} j X_{\beta} \\
X_{\alpha}^{(-)}=\frac{1}{2} X_{\alpha}-\frac{1}{2} j X_{\beta} \\
X_{\beta}^{(+)}=\frac{-1}{2} j X_{\alpha}+\frac{1}{2} X_{\beta} \\
X_{\beta}^{(-)}=\frac{1}{2} j X_{\alpha}+\frac{1}{2} X_{\beta}
\end{gathered}
$$

Since the symmetrical components of the $\alpha$ and $\beta$ are known, the unbalanced two-phase system can be simply represented using (11) and (12).

\section{Practical Implementation of Two-Phase SYSTEMS}

Fig. 11(a) shows the practical implementation of a twophase microgrid system. The two-phase system with voltages in quadrature was implemented with a Danfoss FC302 $2.2 \mathrm{~kW}$ inverter operating at $10 \mathrm{kHz}$ and controlled by a dSPACE DS1103 system. A low-pass-filter (LPF) was implemented with an inductance of $1.5 \mathrm{mH}$ and capacitance of $4 \mu \mathrm{F}$. Both linear and non-linear loads were obtained with the following parameters: $R_{1}=R_{2}=80 \Omega$, and $R_{3}=R_{4}=395 \Omega$, $C_{1}=C_{2}=235 \mu \mathrm{F}$, and $L_{1}=L_{2}=84 \mu \mathrm{H}$ and the results are shown in Fig. 11.

The control system was set up to guarantee sinusoidal voltage waveforms at the power line independently of the load connected to it. The reference phase voltage was defined as $120 V_{r m s}$ and $50 \mathrm{~Hz}$. Resonant controllers [11], [12] were used to regulate the voltages $v_{\alpha}$ and $v_{\beta}$. While Fig. 11(b) shows the two-phase microgrid supplying a linear (Load 1) connected to the line, Fig. 11(c) presents a two-phase microgrid supplying only Load 2. A load transient has also been performed to show the voltage regulation action as presented in Fig. 11(d). In this case it is highlighted a change from a single non-linear load (Load 2) to a combination of Load 1 and Load 2. Notice that the control system is acting satisfactorily.

Fig. 11(e) presents experimental results for the two-phase microgrid supplying the three-leg diode rectifier (Load 3).

\section{CONCLUSION}

In this paper the harmonic analysis of a two-phase diode rectifier system has been analyzed for balanced and unbalanced cases. The analytical model for the harmonic analysis of a two-phase diode bridge rectifier has also been analysed. The results for the simulation and analytical model have been compared to verifiy this method. The mathematical model for the symmetrical components of an unbalanced two-phase system has also been analyzed. 


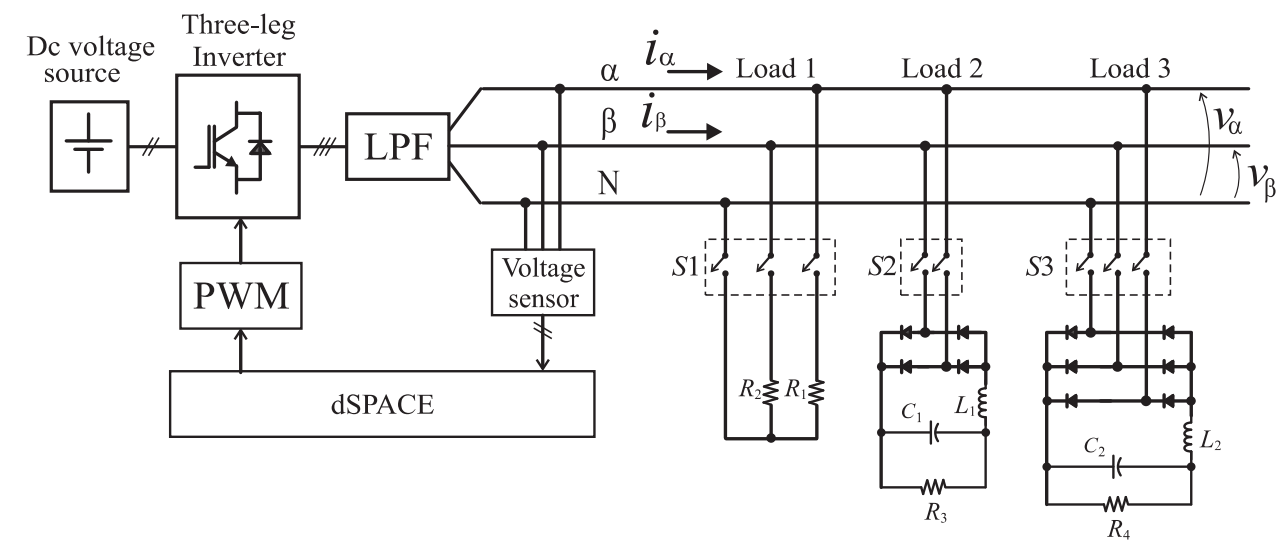

(a)

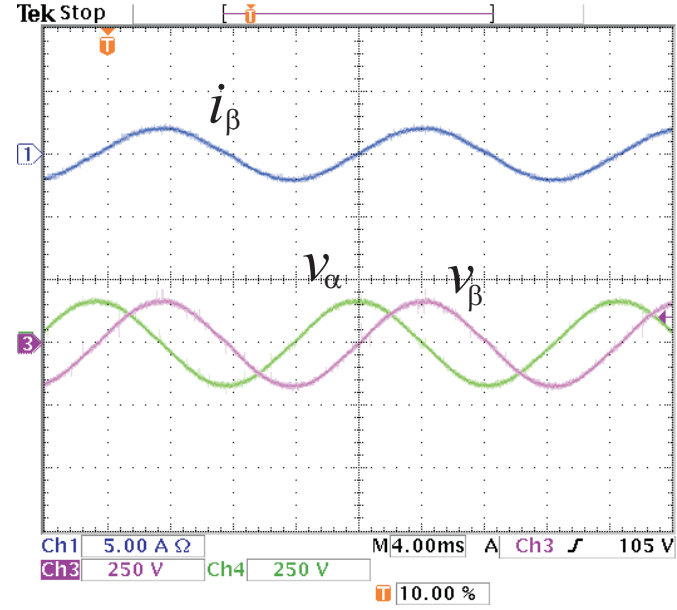

(b)

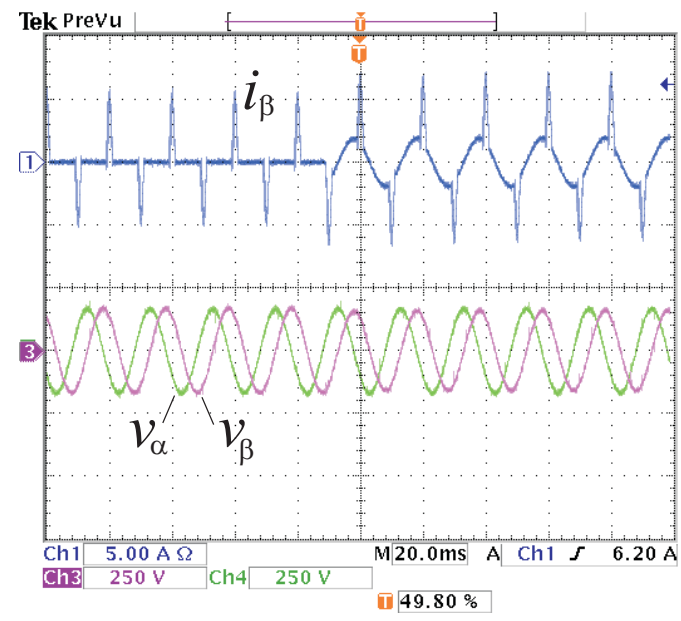

(d)

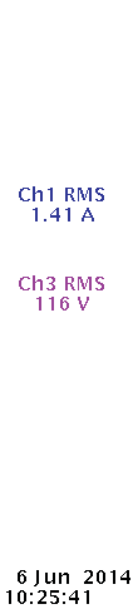

10:25:41

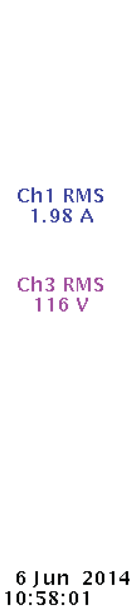

$6 \operatorname{Jun} 2014$
$10: 58: 01$

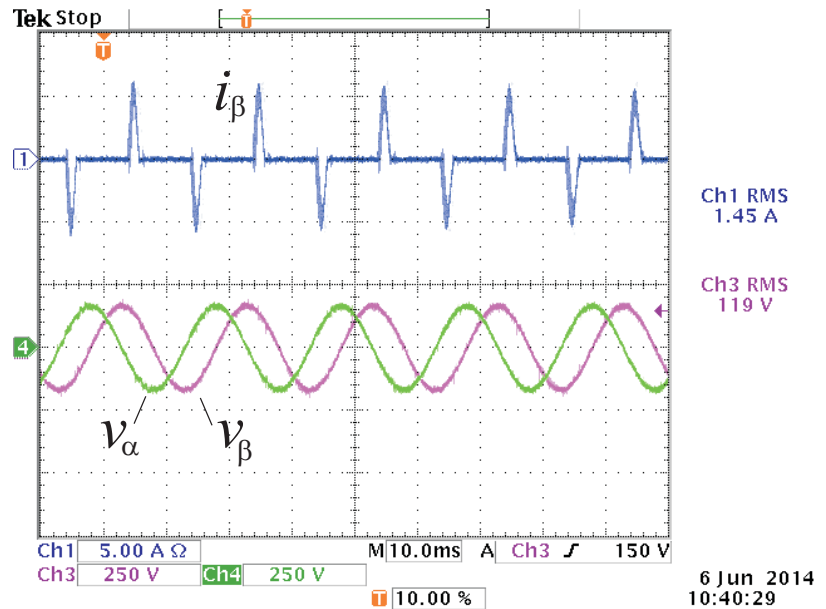

(c)

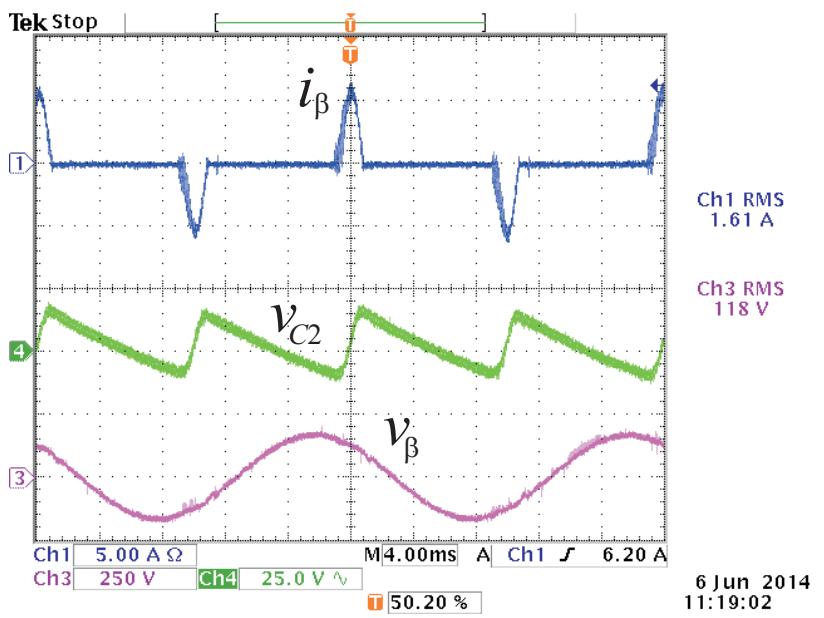

(e)

Fig. 11. (a) Practical implementation of the two-phase microgrid system. (b) Two-phase microgrid supplying a linear load (Load 1). (c) Two-phase microgrid supplying a non-linear load (Load 2). (d) Transient showing a change from the Load 2 to a combination of Loads 1 and 2 . (e) Load current (top), capacitor voltage (middle), and microgrid voltage (bottom).

\section{REFERENCES}

[1] H. Nikkhajoei and R. Iravani, "Steady-state model and power flow analysis of electronically-coupled distributed resource units," IEEE Transactions on Power Delivery, vol. 22, no. 1, pp. 721-728, Jan. 2007.

[2] M. Hamzeh, H. Karimi, and H. Mokhtari, "A new control strategy for a multi-bus mv microgrid under unbalanced conditions," IEEE
Transactions on Power Systems, vol. 27, no. 4, pp. 2225-2232, Nov. 2012.

[3] H. Nikkhajoei and R. Iravani, "Steady-state model and power flow analysis of electronically-coupled distributed resource units," IEEE Transactions on Power Delivery, vol. 22, no. 1, pp. 721 -728, Jan. 2007.

[4] M. Hamzeh, H. Karimi, and H. Mokhtari, "A new control strategy for a multi-bus mv microgrid under unbalanced conditions," IEEE Transactions on Power Systems, vol. 27, no. 4, pp. $2225-2232$, Nov. 
2012.

[5] S. Chakraborty, M. D. Weiss, and M. G. Simoes, "Distributed intelligent energy management system for a single-phase high-frequency ac microgrid," IEEE Transactions on Industrial Electronics, vol. 54, no. 1, pp. $97-109$, Feb. 2007.

[6] Y. W. Li, D. Vilathgamuwa, and P. C. Loh, "A grid-interfacing power quality compensator for three-phase three-wire microgrid applications," IEEE Transactions on Power Electronics, vol. 21, no. 4, pp. 1021 1031, Jul. 2006

[7] Y. Li, D. Vilathgamuwa, and P. C. Loh, "Microgrid power quality enhancement using a three-phase four-wire grid-interfacing compensator," in Proc. of IEEE IAS Meeting '04, vol. 3, Oct. 2004, pp. 1439 - 1446.

[8] E. dos Santos Junior and M. Alibeik, "Microgrid system with voltages in quadrature," in Proc. of IEEE ECCE'13, Sept. 2013, pp. 1344-1349.

[9] K. L. Lian and P. Lehn, "Harmonic analysis of single-phase full bridge rectifiers based on fast time domain method," in Proc. of IEEE ISIE'13, vol. 4, Jul. 2006, pp. 2608-2613.

[10] K. L. Lian, B. Perkins, and P. Lehn, "Harmonic analysis of a three-phase diode bridge rectifier based on sampled-data model," IEEE Transactions on Power Delivery, vol. 23, no. 2, pp. 1088-1096, Apr. 2008.

[11] M. Liserre, R. Teodorescu, and F. Blaabjerg, "Multiple harmonics control for three-phase grid converter systems with the use of pi-res current controller in a rotating frame," IEEE Transactions on Power Electronics, vol. 21, no. 3, pp. 836-841, May 2006.

[12] R. Teodorescu, F. Blaabjerg, M. Liserre, and P. C. Loh, "Proportionalresonant controllers and filters for grid-connected voltage-source converters," IEE Proceedings-Electric Power Applications, vol. 153, no. 5, pp. $750-762,2006$. 